\title{
AN EXTENSION OF MORITA'S METRIZATION THEOREM
}

\author{
CHARLES C. ALEXANDER
}

Abstract. Morita proved that metrizability of a $T_{0}$ space is equivalent to the existence of a sequence of locally finite closed covers which satisfies a refinement condition. We introduce the definition of a cushioned pair-semidevelopment and prove that the existence of a cushioned pair-semidevelopment in a $T_{0}$ space is equivalent to the metrizability of the space. In addition to Morita's theorem, it is seen that several other well-known metrization theorems are also immediate corollaries to the new theorem.

A topological space $X$ is said to be semidevelopable [1] if there is a sequence of (not necessarily open) covers of $X, \gamma=\left\{\gamma_{n}\right\}_{1}^{\infty}$, such that for each $x \in X,\left\{\operatorname{St}\left(x, \gamma_{n}\right)\right\}_{1}^{\infty}$ is a neighborhood base at $x$. In this case, $\gamma$ is called a semidevelopment. If $\gamma$ and $\delta$ are collections of subsets of $X$, then we say that $\gamma$ is cushioned in $\delta$ if one can assign to each $G \in \gamma$ a $D(G) \in \delta$ such that, for every $\gamma^{\prime} \subset \gamma$,

$$
\mathrm{Cl}\left(\cup\left\{G \mid G \in \gamma^{\prime}\right\}\right) \subset \cup\left\{D(G) \mid G \in \gamma^{\prime}\right\} .
$$

By a cushioned pair-semidevelopment for $X$ we shall mean a pair of semidevelopments $(\gamma, \delta)$ such that $\gamma_{n}$ is cushioned in $\delta_{n}$, for each $n$.

REMARKS. (i) If $\gamma=\left\{\gamma_{n}\right\}_{1}^{\infty}$ is a sequence of closure-preserving ${ }^{1}$ closed covers of a space $X$ such that for each $x \in X$ and neighborhood $U$ of $x$, there is an integer $n$ such that $\operatorname{St}\left(x, \gamma_{n}\right) \subset U$, then $(\gamma, \gamma)$ is a cushioned pair-semidevelopment.

(ii) If $X$ is a metric space, $\Sigma_{n}$ is the collection of all spheres of radius less than $1 / n$, for $n=1,2, \cdots$, and $\gamma_{n}$ is a locally finite closed cover of $X$ which refines $\Sigma_{n}$, then $(\gamma, \gamma)$ is a cushioned pair-semidevelopment.

(iii) If $\gamma$ and $\delta$ are open covers of $X$ such that $\gamma^{*}<\delta$, then $\gamma$ is cushioned in $\delta .^{2}$

Received by the editors November 2, 1970.

AMS 1969 subject classifications. Primary 5435; Secondary 5450.

Key words and phrases. Semidevelopable, cushioned pair-semidevelopment, metrizable.

${ }^{1}$ A collection $\gamma$ is said to be closure-preserving if, for every $\gamma^{\prime} C_{\gamma}, \mathrm{Cl}\left(\cup\left\{G \mid G \in \gamma^{\prime}\right\}\right)$ $\subset \cup\left\{\mathrm{Cl}(G) \mid G \in_{\gamma^{\prime}}\right\}$.

${ }^{2}$ If $\gamma$ is a collection of subsets of $X$, then $\gamma^{*}=\{\operatorname{St}(G, \gamma) \mid G \in \gamma\}$, where $\operatorname{St}(G, \gamma)$ $=\cup\left\{H \mid H \in_{\gamma}, H \cap G \neq \varnothing\right\}$. 
Before proceeding to the main theorem we note the following theorem which is due to Michael [6].

Theorem 1. A $T_{1}$ space $X$ is paracompact if and only if every open cover of $X$ has a $\sigma$-cushioned open refinement.

In [6], immediately following the proof of this theorem, Michael comments that it is sufficient to assume that every open cover has a $\sigma$-cushioned refinement, $\gamma=\bigcup\left\{\gamma_{n} \mid n=1,2, \cdots\right\}$, such that the sets $\operatorname{Int}\left(\cup\left\{G \mid G \in \gamma_{n}\right\}\right), n=1,2, \cdots$, cover $X$. The following theorem is a corollary to Theorem 1 and this comment.

Theorem 2. $A T_{1}$ space $X$ is paracompact if and only if every open cover of $X$ has a $\sigma$-cushioned refinement, $\gamma=\bigcup\left\{\gamma_{n} \mid n=1,2, \cdots\right\}$, such that for each $x \in X$ there is an integer $n$ such that $x \in \operatorname{Int} \operatorname{St}\left(x, \gamma_{n}\right)$.

Theorem 3. A space is metrizable if and only if it is $T_{0}$ and has a cushioned pair-semidevelopment.

Proof. The "only if" part of the theorem follows from remark (ii) and the fact that every metrizable space is paracompact.

For the other half of the theorem, assume that $(\gamma, \delta)$ is a cushioned pair-semidevelopment. We can assume that $\gamma_{n+1}<\gamma_{n}$ for each $n$. Because if not, we can replace $\gamma_{n}$ with $\gamma_{n}^{\prime}=\gamma_{1} \wedge \gamma_{2} \wedge \cdots \wedge \gamma_{n}$ for each $n$. Then $\gamma^{\prime}=\left\{\gamma_{n}^{\prime}\right\}_{1}^{\infty}$ is easily seen to be a semidevelopment and since $\gamma_{n}^{\prime}<\gamma_{n}$ for each $n$, each $\gamma_{n}^{\prime}$ is cushioned in $\delta_{n}$. For $G \in \gamma_{n}$, let $D(G) \in \delta_{n}$ be chosen so that the condition of $\gamma_{n}$ being cushioned in $\delta_{n}$ is satisfied.

That $X$ is paracompact follows from Theorem 2 , since a $T_{0}$ semidevelopable space is a $T_{1}$ space. We shall complete the proof by exhibiting a $\sigma$-locally finite basis for $X$ and then applying the NagataSmirnov Theorem [9], [10]. Let $\Sigma_{0}=\{\{x\} \mid x \in X$ and $x$ is an isolated point $\}$. For $n>1$, let $\Sigma_{n}$ be a locally finite open refinement of $\lambda_{n}=\left\{\operatorname{Int} \operatorname{St}\left(x, \gamma_{n}\right) \mid x \in X\right\}$.

Also, if we let

$$
\Sigma_{0, n}=\left\{\{x\} \mid x \in X \text { and } x=\operatorname{St}\left(x, \delta_{n}\right)\right\},
$$

then $\Sigma_{0, n}$ is locally finite, and $\Sigma_{0}=\bigcup\left\{\Sigma_{0, n} \mid n=1,2, \cdots\right\}$. Hence $\Sigma=\bigcup\left\{\Sigma_{n} \mid n=1,2, \cdots\right\} \cup \Sigma_{0}$ will satisfy the Nagata-Smirnov condition, if we can show that it is a basis for $X$.

First define $W_{n}(x)=X \backslash \bigcup\left\{G \mid G \in \gamma_{n}, x \in D(G)\right\}$ for each $x \in X$ and each positive integer $n$. Then, since $\gamma_{n}$ is cushioned in $\delta_{n}$, we have that $x \notin \cup\left\{D(G) \mid G \in \gamma_{n}, \quad x \notin D(G)\right\} \supset \mathrm{Cl}\left(\bigcup\left\{G \mid G \in \gamma_{n}, \quad x \notin D(G)\right\}\right)$ $\supset X \backslash W_{n}(x)$, and therefore, $W_{n}(x)$ is a neighborhood of $x$. Moreover, $\operatorname{St}\left(W_{n}(x), \gamma_{n}\right) \subset \operatorname{St}\left(x, \delta_{n}\right)$ for each $x$ and $n$, since if $G \in \gamma_{n}$ and 
$G \cap W_{n}(x) \neq \varnothing$, then $x \in D(G)$, by definition of $W_{n}(x)$, and hence $G \subset D(G) \subset \operatorname{St}\left(x, \delta_{n}\right)$.

Now assume that $U$ is a neighborhood of $x$, where $x$ is not an isolated point of $X$. Then there is an integer $k$ such that $\operatorname{St}\left(x, \delta_{k}\right) \subset U$, and there is an integer $n$ such that $\operatorname{St}\left(x, \delta_{n}\right) \subset W_{k}(x)$. In fact, we can choose $n>k$, since $X$ is $T_{1}$ and $x$ is not isolated. Then, since $\Sigma_{n}$ is a cover of $X$, there is $S \in \Sigma_{n}$ such that $x \in S$. Then there is $z \in X$ such that $S \subset \operatorname{St}\left(z, \gamma_{n}\right)$, since $\Sigma_{n}<\lambda_{n}$. Thus $x \in \operatorname{St}\left(z, \gamma_{n}\right)$, and therefore, $z \in \operatorname{St}\left(x, \gamma_{n}\right) \subset \operatorname{St}\left(x, \delta_{n}\right) \subset W_{k}(x)$. It follows from the comment above that $\operatorname{St}\left(z, \gamma_{k}\right) \subset \operatorname{St}\left(W_{k}(x), \gamma_{k}\right) \subset \operatorname{St}\left(x, \delta_{k}\right) \subset U$. Hence $x \in S \subset \operatorname{St}\left(z, \gamma_{n}\right)$ $\subset \operatorname{St}\left(z, \gamma_{k}\right) \subset U$, since $\gamma_{n}<\gamma_{k}$. It follows that $\Sigma$ is a basis for $X$.

CoROllary. A space $X$ is metrizable if and only if it is $T_{0}$ and there is a sequence of closure-preserving closed covers of $X, \gamma=\left\{\gamma_{n}\right\}_{1}^{\infty}$, such that for each $x \in X$ and each neighborhood $U$ of $x$, there is an integer $n$ such that $\operatorname{St}\left(x, \gamma_{n}\right) \subset U .^{3}$

Proof. Remark (i) and Theorem 3.

The following theorem is due to Morita [8] and follows immediately from the corollary to Theorem 3 . It should be noted that Theorem 3 can also be proved using Morita's theorem with techniques similar to those in the proof above. However, since Morita's theorem follows trivially from Theorem 3, one can consider Theorem 3 to be an extension of Morita's theorem.

THEOREM 4. A space $X$ is metrizable if and only if it is $T_{0}$ and there is a sequence of locally finite closed covers, $\gamma=\left\{\gamma_{n}\right\}_{1}^{\infty}$, such that for each $x \in X$ and each neighborhood $U$ of $x$, there is an integer $n$ such that $\operatorname{St}\left(x, \gamma_{n}\right) \subset U$.

Theorem 3 may be regarded as a metrization theorem for semistratifiable spaces [4], since they are precisely the spaces which have a $\sigma$-cushioned pair-network ( $=\sigma$-biconservative pair-network) [5].4 Also it may be applied to semimetric spaces, since they are the $T_{0}$ semidevelopable spaces [1].

Incorporated in the next theorem are the statements of several well-known metrization theorems. We will show that in each case the

3 The proof of the necessity of the condition for metrizability in the Corollary is almost obvious and consequently will be omitted. This will also be done for the remaining theorems.

4 A collection, $\gamma$, of ordered pairs of subsets of a space $X$ is a $\sigma$-cushioned pairnetwork if $\gamma=\bigcup\left\{\gamma_{n} \mid n=1,2, \ldots\right\}$, where $\gamma_{n}=\left\{\left(G_{1}^{\alpha}, G_{2}^{\alpha}\right) \mid \alpha \in A_{n}\right\}$, and (i) for any $x \in X$ and any neighborhood $U$ of $x$, there is an integer $n$ and an $\alpha \in A_{n}$ such that $x \in G_{1}^{\alpha} \subset G_{2}^{\alpha} \subset U$ and (ii) $\operatorname{cl}\left(\bigcup\left\{\left.G_{1}^{\alpha}\right|_{\alpha} \in B_{n}\right\}\right) \subset \bigcup\left\{G_{2}^{\alpha} \mid \alpha \in B_{n}\right\}$, for each $n$ and any $B_{n} \subset A_{n}$. 
sufficiency of the condition for metrizability follows easily from Theorem 3.

THEOREM 5. The following conditions are each necessary and sufficient for the metrizability of a space $X$.

(1) (ALEXANDROFF-URYSOHN [2]). $X$ is $T_{0}$ and there is a sequence of open covers, $\left\{\lambda_{n}\right\}_{1}^{\infty}$, such that $\lambda_{n+1}^{*}<\lambda_{n}$ for each $n$, and for each $x \in X$, $\left\{\operatorname{St}\left(x, \lambda_{n}\right)\right\}_{1}^{\infty}$ is a neighborhood base at $x$.

(2) (StONE-ARHANGEL'SKII [11], [3]). $X$ is $T_{0}$ and there is a sequence of open covers, $\left\{\lambda_{n}\right\}_{1}^{\infty}$, such that for each $x \in X$ and each neighborhood $U$ of $x$, there is a neighborhood $V$ of $x$ and an integer $n$ such that $\operatorname{St}\left(V, \lambda_{n}\right) \subset U$.

(3) (MORITA [7]). $X$ is $T_{0}$ and there is a sequence of open covers, $\left\{\lambda_{n}\right\}_{1}^{\infty}$, such that for each $x \in X,\left\{\operatorname{St}^{2}\left(x, \lambda_{n}\right)\right\}_{1}^{\infty}$ is a neighborhood base at $x .^{5}$

Proof. (1) For each $n$, let $\gamma_{n}=\lambda_{2 n}$ and $\delta_{n}=\lambda_{2 n-1}$. In order to see that $(\gamma, \delta)$ is a cushioned pair-semidevelopment, we note that $\gamma_{n}$ is cushioned in $\delta_{n}$ for each $n$, by remark (iii).

(2) Without loss of generality we may assume $\lambda_{n+1}<\lambda_{n}$ for each $n$. Let $\gamma_{n}=\lambda_{n}$ and $\delta_{n}=\lambda_{n}^{*}$. By applying the property of $\left\{\lambda_{n}\right\}_{1}^{\infty}$ twice one sees that $\delta$ is a semidevelopment. It then follows that $(\gamma, \delta)$ is a cushioned pair-semidevelopment.

(3) See proof of (2).

\section{BIBLIOGRAPHY}

1. C. Alexander, Semi-developable spaces and quotient images of metric spaces, Pacific J. Math. (to appear).

2. P. Alexandroff and P. Urysohn, Une condition necessaire et suffisante pour qu'une classe (L) soit une classe (D), C. R. Acad. Sci. Paris 177 (1923), 1274-1276.

3. A. V. Arhangel'skit, New criteria for the paracompactness and metrizability of an arbitrary $T_{1}$-space, Dokl. Akad. Nauk SSSR 141 (1961), 13-15=Soviet Math. Dokl. 2 (1961), 1367-1369. MR 24 \#A1113.

4. G. Creede, Semi-stratifiable spaces, Topology Conference, Arizona State University, Tempe, Ariz., 1967, pp. 318-323.

5. Ja. J. Kofner, A new class of spaces and some problems of symmetrizability theory, Dokl. Akad. Nauk SSSR 187 (1969), 270-273=Soviet Math. Dokl. 10 (1969), 845-848. MR 40 \#1964.

6. E. A. Michael, Yet another note on paracompact spaces, Proc. Amer. Math. Soc. 10 (1959), 309-314. MR 21 \#4406.

7. K. Morita, On the simple extension of a space with respect to a uniformity. IV, Proc. Japan Acad. 27 (1951), 632-636. MR 14, 571.

- If $\lambda$ is a collection of subsets of $X, x \in X$, and $n$ is a positive integer, we have

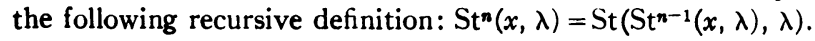


8. - A condition for the metrizability of topological spaces and for n-dimensionality, Sci. Rep. Tokyo Kyoiku Daigaku Sect. A 5(1955), 33-36. MR 17, 179.

9. J. Nagata, On a necessary and sufficient condition of metrizability, J. Inst. Polytech. Osaka City Univ. Ser. A Math. 1 (1950), 93-100. MR 13, 264.

10. Yu. M. Smirnov, A necessary and sufficient condition for metrizability of a topological space, Dokl. Akad. Nauk SSSR 77 (1951), 197-200. MR 12, 845.

11. A. H. Stone, Sequences of coverings, Pacific J. Math. 10 (1960), 689-691. MR 22 \#955.

University of Virginia, Charlottesville, Virginia 22904

University of MisSISSIPPI, UNIVERSITy, MisSISSIPPI 38677 